\title{
A modular RNA interference system for multiplexed gene regulation
}

\author{
Ari Dwijayanti, Marko Storch ${ }^{1}$, Guy-Bart Stan ${ }^{1,2,{ }^{*}}$ and Geoff S. Baldwin ${ }^{\oplus 1,3, *}$ \\ ${ }^{1}$ Imperial College Centre for Synthetic Biology, Imperial College London, London SW7 2AZ, UK, ${ }^{2}$ Department of \\ Bioengineering, Bessemer Building, South Kensington Campus, Imperial College London, London SW7 2AZ, UK and \\ ${ }^{3}$ Department of Life Sciences, Sir Alexander Fleming Building, South Kensington Campus, Imperial College London, \\ London SW7 2AZ, UK
}

Received August 07, 2020; Revised December 20, 2021; Editorial Decision December 20, 2021; Accepted December 22, 2021

\begin{abstract}
The rational design and realisation of simple-to-use genetic control elements that are modular, orthogonal and robust is essential to the construction of predictable and reliable biological systems of increasing complexity. To this effect, we introduce modular Artificial RNA interference (mARi), a rational, modular and extensible design framework that enables robust, portable and multiplexed post-transcriptional regulation of gene expression in Escherichia coli. The regulatory function of mARi was characterised in a range of relevant genetic contexts, demonstrating its independence from other genetic control elements and the gene of interest, and providing new insight into the design rules of RNA based regulation in E. coli, while a range of cellular contexts also demonstrated it to be independent of growth-phase and strain type. Importantly, the extensibility and orthogonality of mARi enables the simultaneous posttranscriptional regulation of multi-gene systems as both single-gene cassettes and poly-cistronic operons. To facilitate adoption, mARi was designed to be directly integrated into the modular BASIC DNA assembly framework. We anticipate that mARi-based genetic control within an extensible DNA assembly framework will facilitate metabolic engineering, layered genetic control, and advanced genetic circuit applications.
\end{abstract}

\section{INTRODUCTION}

Synthetic biology aims to make the engineering of biological systems more predictable, efficient and reliable (1-3). With this goal in mind, our ability to pre- dictably combine genetic parts into higher-level functional biological/biochemical systems of increasing complexity is essential to improve the efficiency of the Design-BuildTest-Learn cycle $(4,5)$. In this work, we focus on expanding the toolbox of modular biomolecular control elements that function at the post-transcriptional level, specifically small non-coding RNAs (sRNAs). The ubiquity of sRNAbased control in critical cellular processes such as homeostasis and adaptation demonstrates their importance for the design and control of biological systems (6-10). The sRNAs act to coordinate and synchronise multiple signals through sequence-specific and transient RNA-RNA interactions $(11,12)$, typically leading to down-regulation of target gene expression (13-16).

Based on the arrangement of sRNAs and their targets, sRNAs can be grouped into cis- and trans-encoded sRNAs. The cis-encoded sRNAs are transcribed from the same loci as their target mRNA, but as a reverse complement, thus making a complementary base-pairing to the mRNA they regulate; in contrast, trans-encoded sRNAs are located in a distal site. Despite this, trans-encoded sRNAs can have efficient regulation of their mRNA targets (14,17-19). In bacterial systems, trans-encoded sRNAs rather than cisencoded sRNAs have been reported to be especially effective in silencing gene expression due to their longer halflife in the cytoplasm, thought to be due to association with the Hfq chaperone protein (20-23). The trans-encoded sRNAs, therefore, have been widely applied as versatile genetically encoded controllers for the design of synthetic biological circuits for metabolic engineering and synthetic biology purposes $(7,24-27)$.

Previous approaches to implement trans-encoded sRNA were exemplified by the use of native sRNAs and their cognate targets, i.e. $r p o S, h n s, \operatorname{sodB}$ and $\operatorname{smp} C(28,29)$. By fusing the leader sequences from these natural sRNA systems to a reporter gene (e.g. GFP and mCherry), the performance of the native sRNA system could be quantified from the fluo-

\footnotetext{
${ }^{*}$ To whom correspondence should be addressed. Tel: +44 207594 5288; Email: g.baldwin@imperial.ac.uk

Correspondence may also be addressed to Guy-Bart Stan. Email: g.stan@imperial.ac.uk

Present addresses:

Ari Dwijayanti, CNRS@CREATE, 1 Create Way, \#8-01 Create Tower, Singapore 138602.

Marko Storch, London Biofoundry, Translation \& Innovation Hub, White City Campus, Imperial College London, London W12 0BZ, UK.
}

(C) The Author(s) 2022. Published by Oxford University Press on behalf of Nucleic Acids Research.

This is an Open Access article distributed under the terms of the Creative Commons Attribution License (https://creativecommons.org/licenses/by/4.0/), which permits unrestricted reuse, distribution, and reproduction in any medium, provided the original work is properly cited. 
rescence output. Due to its highly composable structure, the sRNA can also be re-programmed to target any RNA sequence by modification of the seed sequence, whilst retaining the native sRNA scaffold $(17,18,28)$. We term this new type of sRNA an artificial sRNA system. Several rules and guides have been developed to improve the rational design of a set of novel sRNA systems $(17,27,30,31)$. This strategy has been implemented to create artificial sRNA systems with bespoke designs, where the sRNA is targeted to bind the first $24 \mathrm{bp}$ of the GOIs $(25,32)$. Artificial sRNAs have also been employed to specifically bind a set of standardised target sequences that are inserted upstream of GOIs (24). However, the requirement to insert specific target sites upstream of each GOI may impede the implementation and adoption of sRNA-based controllers for broader applications.

To leverage the use of sRNAs in genetic circuits constructed via a modular DNA assembly method, we set out to design a universal, modular framework for the facile implementation of trans-acting sRNA-based control using verified, re-usable target sequences, namely modular Artificial RNA interference (mARi). BASIC DNA assembly provides a modular, standardised and automatable (33) framework for genetic structure based on orthogonal, computationally designed linkers (34) (Figure 1). We exploited this by targeting the DNA linkers used in the construction of gene expression cassettes and operons. In our mARi design, a modified seed sequence specific to the linker upstream of the target Gene of Interest (GOI) was fused to a native sRNA scaffold containing a host factor-1 (Hfq) binding site $(17,27)$ (Figures 1A and 2A). The integration of mARi-based regulation into a modular DNA assembly method offers a simple yet powerful strategy for implementing sRNA regulatory systems. Since standardised linkers are used in the assembly process, gene expression can be controlled by expression of an mARi that is cognate to the linker sequence upstream of the target gene (Figure 1). We characterise the post-transcriptional regulation of $\mathrm{mARi}$ in the context of various genetic design parameters including transcript ratio, molecular copy number, spatial organisation, growth temperatures and media, host strain, and growth phase, thus expanding our understanding of the design rules for new artificial sRNA-based regulators. Finally, mARi sequence variants were further expanded and their implementation was demonstrated for simultaneous regulation in a multi-gene system with different genetic architectures.

\section{MATERIALS AND METHODS}

\section{Bacterial strains and outgrowth conditions}

The following Escherichia coli strains were used for plasmid construction and expression: $\mathrm{DH} 5 \alpha\left(\mathrm{F}^{-}\right.$endAl glnV44 thi1 recAl relA1 gyrA96 deoR nupG purB20 $\varphi 80 \mathrm{~d} l a c Z \Delta \mathrm{M} 15$ $\triangle($ lacZYA-argF $) \mathrm{U} 169$, hsdR17 $\left.\left(r_{K}{ }^{-} m_{K}{ }^{+}\right), \lambda^{-}\right)$, DH10b [F-

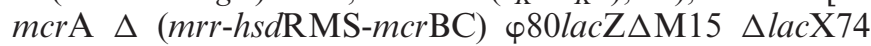
recA1 end $\mathrm{A} 1$ ara $\mathrm{D} 139 \Delta$ (ara leu) 7697 gal $\mathrm{U}$ galK rpsL nup $\mathrm{G} \lambda-]$, BL21 (DE3) ( $\mathrm{F}^{-}$omp $\mathrm{T} h s d \mathrm{~S}_{\mathrm{B}}\left(\mathrm{r}_{\mathrm{B}}{ }^{-}, \mathrm{m}_{\mathrm{B}}{ }^{-}\right)$gal dcm (DE3)) and BL21star (DE3) $\left(\mathrm{F}^{-}\right.$omp $\mathrm{T} h s d \mathrm{~S}_{\mathrm{B}}\left(\mathrm{r}_{\mathrm{B}}^{-}, \mathrm{m}_{\mathrm{B}}{ }^{-}\right)$ gal dcm rne131 (DE3)). E. coli colonies were grown from single-colony isolates in LB (Luria Bertani) medium supplemented with the appropriate antibiotics, shaken at 220 rpm and grown at $37^{\circ} \mathrm{C}$ (unless specified otherwise). The antibiotics used for plasmid maintenance were carbenicillin and kanamycin at a concentration of 100 and $50 \mu \mathrm{g} / \mathrm{ml}$, respectively.

\section{Design and analysis of mARi sequences}

The sequences of UTR-RBS linkers used in BASIC DNA assembly (34) were designed and validated using R2o DNA designer (35). Secondary structures of the mARi sequences were predicted using RNAFold WebServer (http: //rna.tbi.univie.ac.at/cgi-bin/RNAWebSuite/RNAfold.cgi) and visualized using VARNA GUI (36) (http://varna.lri. fr/index.php?lang=en\&page=tutorial\&css=varna). $\quad$ The predicted interactions between mARi regulators and their mRNA targets were simulated through IntaRNA $(37,38)$ (https://bio.tools/intarna). The free binding energy of mARi and its mRNA target site was estimated using a web-based service of the Two-State Melting Hybridisation of UNAFold RNA folding software DINAMelt (39) (http://www.unafold.org/Dinamelt/applications/ two-state-melting-hybridization.php) using default parameters at $37^{\circ} \mathrm{C}$. The percent identity of mARi-UTR pairs was calculated using the EMBOSS needle method (https://www.ebi.ac.uk/Tools/psa/emboss_needle/). The Hamming distance was calculated using a custom Python script (https://github.com/MsDwijayanti/hamming/blob/ main/hamming.py). The predicted expression strength of the UTR-RBS linker was simulated using EMOPEC (40) and RBS calculator v2.0 $(41,42)$ with the input sequence starting from the Transcription Start Site $(+1)$ to $100 \mathrm{bp}$ of the downstream CDS. The off-target effect was computed using CopraRNA $(38,43,44)$ (https://bio.tools/coprarna). NC_000913 (E. coli MG1655), NC_010473 (E. coli DH10b), NC_012892 and NC_012971 (E. coli BL21 (DE3)) were used as inputs references for host-background off-target prediction.

\section{Plasmid assembly}

Plasmids were constructed using the BASIC DNA assembly method $(34,45)$. New DNA parts with prefix and suffix sequences were synthesised as gBlocks by Integrated DNA Technologies (IDT), gene fragments by TWIST Bioscience, or derived by PCR mutagenesis. The gBlocks and gene fragments were directly cloned into pJET1.2 or pUC AmpR_plasmid as BASIC bioparts. PCR mutagenesis for generating new BASIC parts were carried out using Phusion Polymerase (NEB) and phosphorylated oligonucleotides (IDT). Sequence verification of BASIC bioparts was carried out using Sanger sequencing (Source Bioscience). Details of the BASIC bioparts used to construct the plasmids are provided in Supplementary Table S5. Linkers for BASIC assembly were provided by Biolegio or IDT (Supplementary Table S6). All constructs were transformed into chemically competent $E$. coli DH5 2 . Maps of plasmids used in this study are provided in Supplementary Figure S9. 


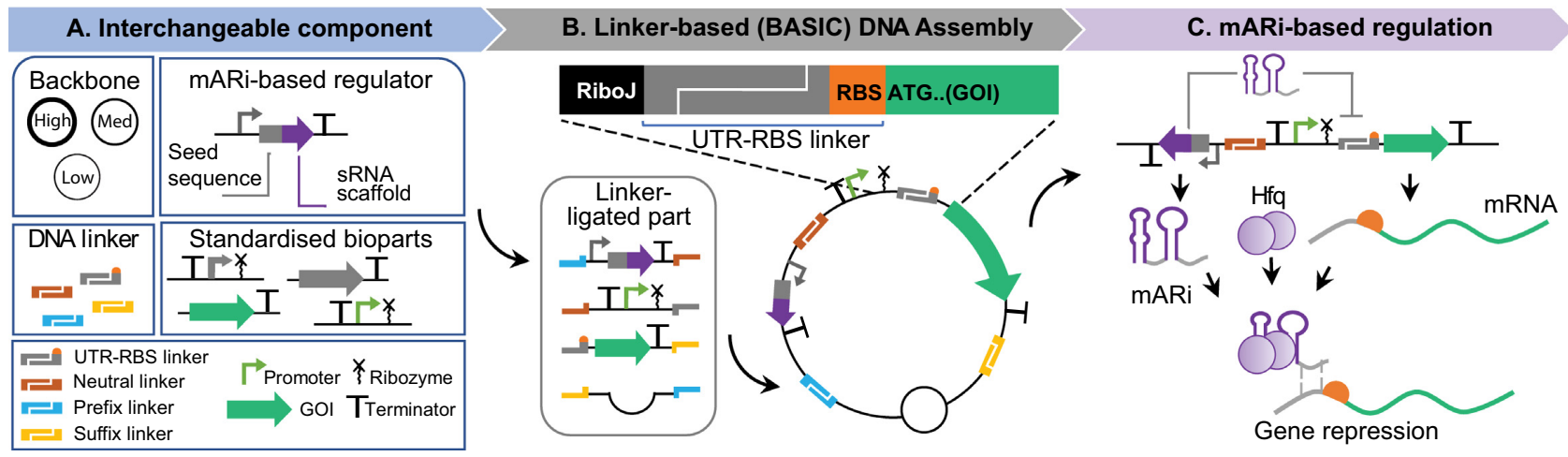

Figure 1. mARi-based regulation integrated into the BASIC modular design and assembly framework. (A) mARi-based regulators composed of a promoter, seed sequence (grey), sRNA scaffold (purple) and terminator. These regulators were created as interchangeable components together with standardised DNA parts (i.e. plasmid backbone, promoter, and coding sequence) and computationally designed DNA linkers (i.e. neutral, UTR-RBS, and Prefix/Suffix linker) used in the BASIC DNA assembly framework (34). (B) Plasmids were constructed from the interchangeable DNA parts using BASIC DNA assembly: a representative assembled plasmid shows a functional UTR-RBS linker controlling expression of a downstream gene of interest (GOI). (C) Expression of mARi leads to repression of a target gene with a cognate UTR by base-pairing between the 5'untranslated region and the mARi seed sequence.

\section{Flow cytometry assay}

Cellular expression of sfGFP and mCherry was performed in 96-well plates with biological triplicates for each construct. E. coli carrying an empty backbone (containing only an origin of replication and an antibiotic resistance gene) was used as a negative control. E. coli colonies were inoculated from glycerol stock into LB media supplemented with appropriate antibiotics. The colonies were grown for $16 \mathrm{~h}$ at $30^{\circ} \mathrm{C}$ and $600 \mathrm{rpm}$ in a benchtop shaker (Mikura). The culture was then diluted 200x $(10 \mu$ into $190 \mu$, then 10 $\mu 1$ into $90 \mu 1$ ) in LB media using an automated liquid handling robot (CyBio Felix). The plate was grown at $37^{\circ} \mathrm{C}$ and $600 \mathrm{rpm}$ in a benchtop shaker (Mikura). After $6 \mathrm{~h}$ of incubation, the cell density was measured by absorption at 600 $\mathrm{nm}\left(\mathrm{Abs}_{600}\right.$; not normalised for path length). Abs 600 typically reached values in the range $0.6-0.7$. Two microliters of liquid culture were taken and diluted into $200 \mu 1$ Phosphate Buffer Saline (PBS) supplemented with $2 \mathrm{mg} / \mathrm{ml}$ kanamycin to inhibit further protein synthesis and cell growth. The fluorescence data of single cells for the dual reporters system (Figure 4B and D) was collected using BD Fortessa flow cytometer with a $488 \mathrm{~nm}$ excitation laser and filter 530/30 for sfGFP; $561 \mathrm{~nm}$ excitation laser and filter 610/20 for mCherry. For the triple reporters system (Figure 4C), fluorescence was measured using Attune NxT flow cytometer with a $488 \mathrm{~nm}$ excitation laser and channel BL1 530/30 for sfGFP; $561 \mathrm{~nm}$ excitation laser and channel YL2 620/15 for mCherry; $405 \mathrm{~nm}$ excitation laser and channel VL1 440/50 for mTagBFP. In total 10,000 events were collected for each sample. The data was stored as an FCS file 3.0 and analysis was done using Flow Jo V10. Single cell population gating was performed after plotting FSC-H against SSC-H and histograms of each channel of fluorescence. The chosen gating covered $86-99 \%$ of the total population. The fluorescence intensity of the sample was calculated by subtracting the geometric mean fluorescence of the control (strains with empty backbone). The normalised fluorescence was calculated by dividing the background-subtracted mean fluorescence of the sample with mARi by the background- subtracted mean fluorescence of the sample without mARi expression.

\section{Plate reader assay}

For continuous growth assay, overnight liquid cultures (as for flow cytometry assay) were diluted $200 \times$ in LB media (10 $\mu$ l into $190 \mu \mathrm{l}$, then $10 \mu \mathrm{l}$ into $90 \mu \mathrm{l}$ ) using an automated liquid handling robot (CyBio Felix); the plate was then incubated in a microplate reader (Clariostar, BMG Labtech) with continuous shaking at $37^{\circ} \mathrm{C}$ and $600 \mathrm{rpm}$ for $12-24 \mathrm{~h}$. For different growth temperatures, the DH $5 \alpha$ cells with and without $\mathrm{mARi}$ expression plasmids were incubated at $30^{\circ} \mathrm{C}$ or $37^{\circ} \mathrm{C}$ in LB medium. For different growth media, the engineered DH5 $\alpha$ cell were tested in two mediums: LB and EZ MOPS RDM supplemented with $0.2 \%$ glucose. For the EZ MOPS RDM media supplemented with $0.2 \%$ glucose, 5 $\mu 1$ samples were diluted into $95 \mu \mathrm{l}$ of fresh media using an automated liquid handling robot (CyBio Felix). The plate was incubated at $37^{\circ} \mathrm{C}$ and $600 \mathrm{rpm}$.

Absorbance at $600 \mathrm{~nm}$ and GFP fluorescence (F:48216/F:530-40) were measured every $15 \mathrm{~min}$. The corrected $\mathrm{Abs}_{600}$ was calculated by subtracting the mean $\mathrm{Abs}_{600}$ of media only control from the $\mathrm{Abs}_{600}$ of each well. The fluorescence intensity was corrected by subtracting the mean fluorescence value of negative control at equivalent $\mathrm{Abs}_{600}$. The value $\mathrm{Fl} / \mathrm{Abs}_{600}$ was calculated by dividing the value of corrected fluorescence by corrected $\mathrm{Abs}_{600}$. Statistical analysis was calculated in Prism v.8.0 (GraphPad). The values were compared using two-tailed Student's t-test for unpaired comparisons and one-way ANOVA.

\section{RESULTS}

Design of a modular Artificial RNA interference regulation system

The mARi sequences were designed with two core components: the seed sequence and an sRNA scaffold containing the $\mathrm{Hfq}$ binding site (Figures $1 \mathrm{~A}$ and 2A). The 
A

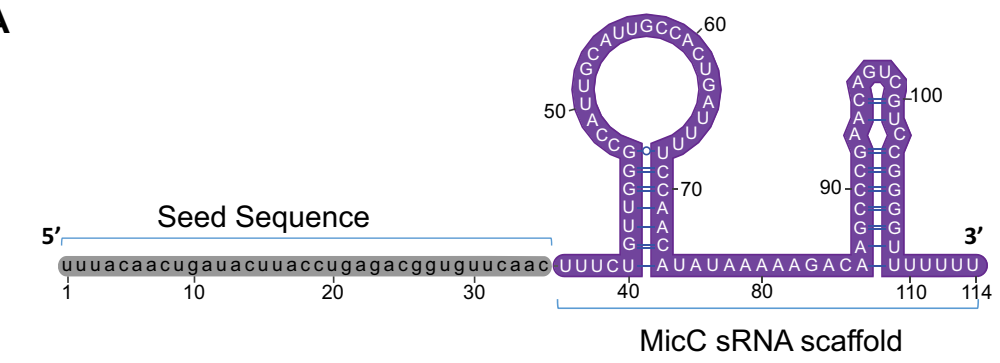

C Without mARi With mARi

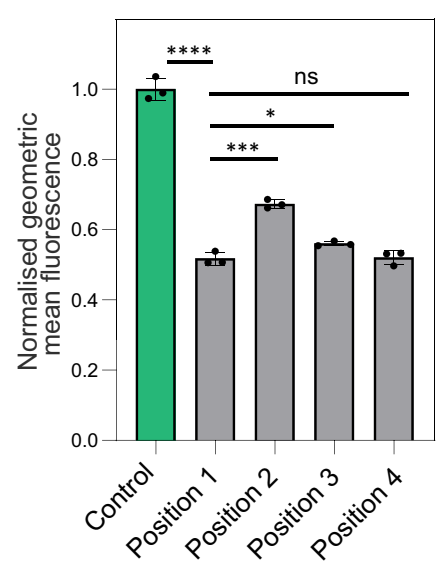

D

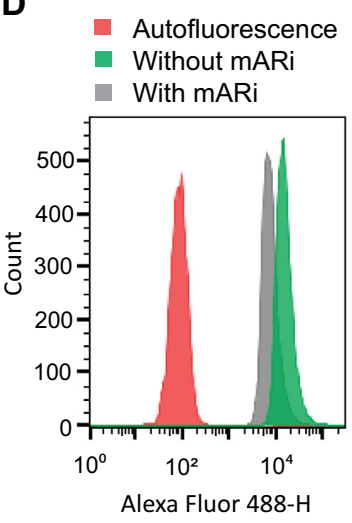

B

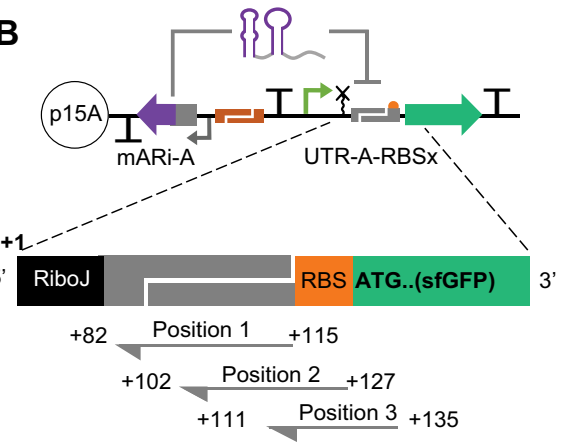

E

Figure 2. Design and development of mARi-based gene regulation. (A) Sequence and secondary structure of mARi containing a seed sequence (grey) and MicC RNA scaffold (purple). (B) Schematic design of the target site selection schema for mARi-mediated repression. Four positions within the translation initiation region were selected as target sites (Positions 1-4; Supplementary Table S1). The numbers indicate the relative position of mARi downstream of the transcription start site $(+1)$. (C) Functional characterisation of mARi-based gene regulation showing the silencing activity of mARi-A for the different target positions vs a sfGFP-only control without mARi. (D) Uniformity of representative expressing strains with and without $\mathrm{mARi}$ expression. (E) The fluorescence intensity (top) and normalized fluorescence (bottom) when position $1 \mathrm{mARi}$-A was used to target UTR-A with varying RBS strengths. All sfGFP fluorescence measurements were performed by flow cytometry after $6 \mathrm{~h}$ growth. Data are shown with error bars for the mean \pm SD of triplicate measurements (black dots). Statistically significant differences were determined using two-tailed Student's $t$-test (**** represents $P<0.0001$, $* * *$ represents $P<0.001$, * represents $P<0.1$, ns represents not significant).

scaffold sequence is responsible for recognition by the RNA chaperone protein Hfa, which is highly conserved as part of natural cellular regulation $(46,47)$ in a wide range of organisms (48-51). One of the intensively-studied Hfq-dependent sRNAs, MicC was chosen from sRNA scaffolds naturally found in E. coli (52) and Salmonella enterica (16). The inclusion of this RNA chaperone binding site in the sRNA structure has previously been shown to one of the best $\mathrm{Hfq}$ binding sites to mediate sRNA-based gene repression (30). The nature of the multiple targets of MicC based sRNAs in controlling the expression of outer membrane proteins $(\mathrm{OmpC}$ and $\mathrm{OmpD}$ ) can be exploited to target any mRNA sequence of interest. Based on this architecture, the seed sequences can be modified while preserving the native $\mathrm{MicC}$ scaffold $(17,25,27,30)$ (Figure 2A).

The seed sequence within mARi creates a specific basepairing interaction to a target sequence in the mRNA of interest, resulting in its post-transcriptional silencing. To standardise and enhance the modularity of mARi, the seed sequence was designed to target expression cassettes constructed via BASIC DNA assembly (34). BASIC assembly relies on standardised linkers between interchangeable DNA parts (Figure 1A) (34). Expression cassettes and operons can be tuned using functional DNA linkers that encode a ribosome binding site (RBS) within a defined $5^{\prime}$ untranslated region (UTR; UTR-RBS linker; Figure 1B). Since BASIC UTRs are computationally designed to be orthogonal to the host genome and exclude secondary structure (35), they provide an ideal target for mARi and reduce offtarget effects.

We designed mARi sequences to target the translation initiation region of mRNA for three main reasons: (i) the $5^{\prime}$ UTR sequences are relatively AU rich (30-43.8\% GC; Supplementary Table S1) and AU rich sequences are 
preferentially bound by $\mathrm{Hfq}$ (53); (ii) targets located in the translation initiation region have been shown to exhibit a low off-target effect in trans-encoded sRNAbased regulation (30), which is important for regulator specificity/orthogonality; (iii) a $5^{\prime}$ UTR target site location offers potential modularity since it is independent of different GOIs and is compatible with the modular UTRRBS linkers in the BASIC design framework (34). To evaluate this approach, seed sequences were designed to target different positions in the translation initiation region (Figure 2B). Positions 1-3 addressed gene-independent targets within the BASIC framework, while position 4 was specific to the gene of interest, in this case superfolder-gfp ( $s f g f p$; Figure 2B). The reverse complements of the cognate target sequences were combined with the MicC scaffold to create a series of mARi sequences (Supplementary Table S1).

The design of the full sequence of each mARi variant was computationally evaluated to ensure that it met the requirements for effective repression (30). Firstly, the designed sequence for each $\mathrm{mARi}$ was made compatible with BASIC DNA assembly (34), specifically avoiding the forbidden restriction site $B s a \mathrm{I}$. Secondly, to achieve effective repression and to have a high affinity binding between the seed and target sequences, the GC content and binding energy of the seed sequences were calculated and found to vary from $34.62 \%$ to $43.8 \%$ and -38.6 to $-61 \mathrm{kcal} / \mathrm{mol}$, respectively. The binding energy has a positive correlation to the binding affinity and observed repression capability $(27,30)$, and previous studies suggested a typical binding energy of -30 to $-40 \mathrm{kcal} / \mathrm{mol}(17,27)$ was required for effective repression activity. However, lower binding energy (less than $-40 \mathrm{kcal} / \mathrm{mol}$ ) sRNAs have been experimentally validated and suggested to achieve higher repression (30). Homology length determines mARi specificity and target discrimination (30): shorter binding sites have a lower binding affinity and may cause off-target effects $(17,27)$, while longer binding sequences may have a complex secondary structure that could cause binding to the mRNA target to be less thermodynamically favorable (30). All mARi regulators used here were designed to have perfect complementarity in their seed sequences to their target mRNA. (Supplementary Figure $\mathrm{S} 1 \mathrm{~b})$.

To investigate the repression efficiency of mARi targets, sfGFP expression cassettes were assembled with a constitutive promoter $\mathrm{P}_{\mathrm{J} 23101 \text { BASIC }}$ and UTR-A-RBSc linker, while mARi expression was driven by the constitutive $\mathrm{P}_{\mathrm{J} 23101}$ (Figure 1B). Measurement of reporter expression demonstrated that all mARi target sequences led to repression of sfGFP, compared to control cells lacking mARi (Figure 2C). The intra-gene target at position 4 showed significant repression as anticipated $(17,25,27)$. However, targeting this position would require bespoke design of a specific sequence for the target gene, thus optimisation for each new expression target is required; a less desirable strategy from a modular engineering perspective. Position 1, which targets the UTR upstream of the RBS was as effective as position 4 (Figure 2C). The position 1 target shown had been further optimised for length; reducing the length to $25 \mathrm{bp}$, as shown for the other positions, had only a minor impact, although further reduction to $20 \mathrm{bp}$ resulted in significant loss of repression activity (Supplementary Figure S2). Targeting this standardised
UTR component of the BASIC framework is ideal for modular engineering since it is independent of both the GOI and RBS. The homogeneity of representative producing strains with mARi position 1 against the control strains without mARi expression was shown in Figure 2D.

To evaluate the independence of position 1 UTR target, a set of constructs with five different RBS strengths (RBSaRBSe) (Supplementary Table S2) controlling sfGFP translation were assembled both with and without the position 1 mARi cassette all within the same UTR-A context. The $s f g f p$ gene-only constructs resulted in a series of fluorescence intensities reflecting RBS strength variation (Figure 2E). The position 1 UTR-A targeted mARi cassette reduced the fluorescence signal in all cases and the relative repression was found to be constant across the series (Figure 2E). This demonstrated that the repression activity of mARi-A at position 1 was independent of RBS strength, an important feature for RBS design flexibility and modularity of mARi.

\section{mARi activity is tuneable via genetic design parameters}

To evaluate the features of mARi, we characterised its responsiveness to genetic design parameters and its robustness to different contexts and growth conditions. Two key determinants modulating the repression activity of this post-transcriptional regulator are (i) the degradation rate of RNA complexes and (ii) the ratio of transcript abundance (54). We focused on investigating the relative abundance of $\mathrm{mARi}$ in relation to its target mRNA. Unlike previous work where inducible expression was used to modulate relative transcript abundance of sRNA to mRNA target $(13,32,54)$, here we constitutively expressed both transcripts to test the impact of different transcript levels, which provides more consistent relative expression. Relative transcript abundance of mARi and mRNA was estimated from the relative promoter strength driving both regulator and target (54). A set of standardised constitutive promoters ( $\left.\mathrm{P}_{\mathrm{J} 23 x x x \_B A S I C}\right)$ were used to express a $s f G F P$ reporter gene using UTR A-RBSc in a p15A backbone (Supplementary Figure S3a). The functional characterisation results were then used as a basis to infer the relative transcript expression ratio (Figure $3 \mathrm{~A}$ ), which is calculated by dividing the strength of the promoter used for mARi by the promoter expressing the mRNA target. To assess the impact of the mARi:mRNA ratio, a matrix of designs with four different promoter strengths for both mARi and mRNA were constructed (Figure 3A, B). The resulting sfGFP expression showed a consistent trend, with sfGFP fluorescence decreasing as the mARi:mRNA ratio increased (Figure 3C, D, Supplementary Figure S3c, Supplementary Table S3). The maximum repression was observed when an excess of $\mathrm{mARi}$ was present in relation to its target mRNA, presumably because an excess of $\mathrm{mARi}$ is required to saturate binding of target mRNA. On the other hand, a low expression ratio led to weak repression, presumably due to an insufficient amount of mARi being available to inactivate mRNA target $(30,54)$.

A further variable affecting intracellular transcript abundance is plasmid copy number used in the system. Designs with both high (blue box in Figure 3A) and low (grey box in Figure 3A) expression ratios were selected to exemplify dif- 

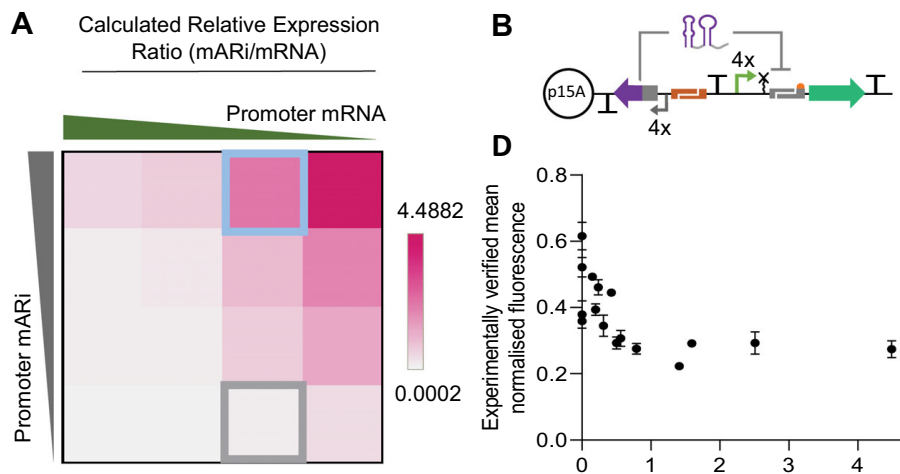

D

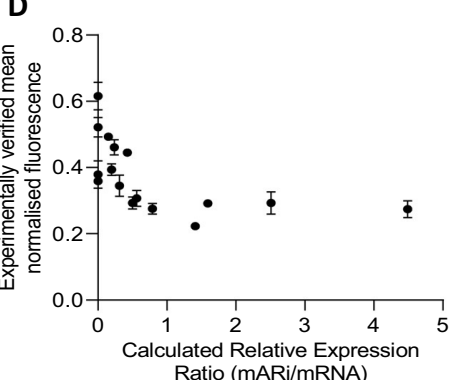

Ratio (mARi/mRNA)

E

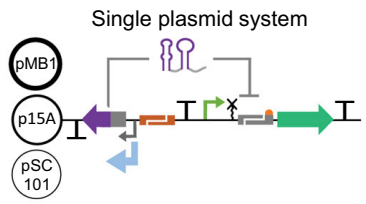

F
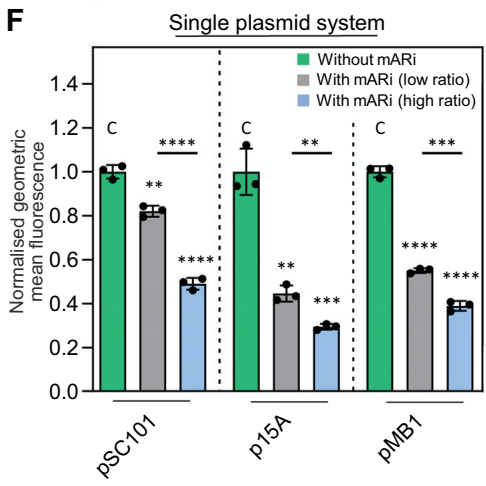

K
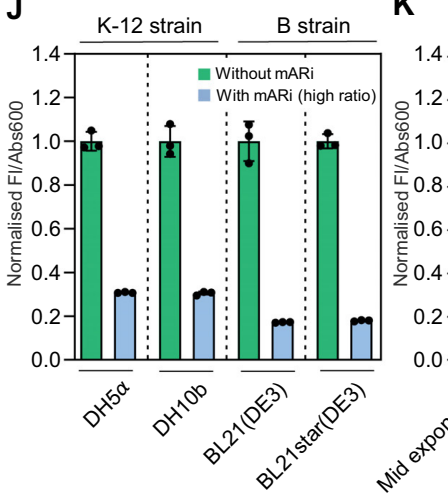

DH10b
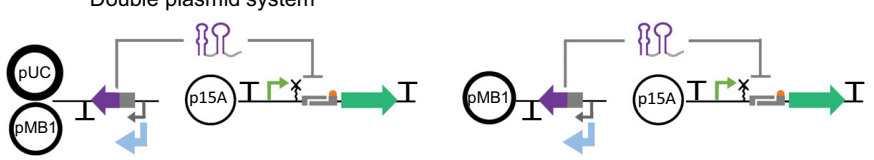

G
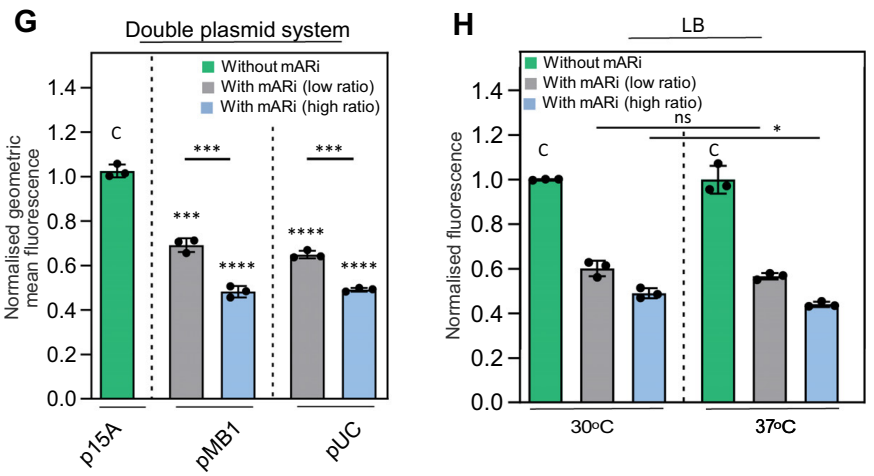
Experimentally verified mean
normalised fluorescence
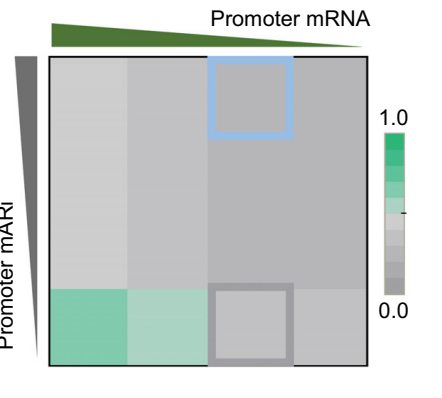

Figure 3. Characterisation of mARi-A across different genetic architectures and growth conditions. (A) Heat-map showing relative expression ratio calculated from the relative strength of promoters driving mARi and mRNA expression across a matrix of constructs (Supplementary Figure S3b). The representative conditions used for set-point high and low expression ratios are highlighted with a blue square $\left(\mathrm{P}_{\mathrm{J} 23119}-\mathrm{mARi}_{\mathrm{J}} \mathrm{P}_{\mathrm{J} 23101 \mathrm{BASIC}}-\mathrm{mRNA}\right)$ and

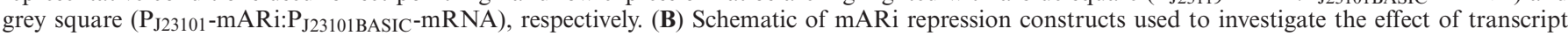
ratios using four different strength constitutive promoters driving both mARi and target mRNA in a single plasmid system. (C) Heat-map showing the measured relative expression levels (mean normalized fluorescence) of the mARi expression ratio matrix from (A). The calculated relative expression ratio and repression activity of mARi are provided in Supplementary Table S3. (D) Correlation of transcript expression ratios in (A) and normalised fluorescence in (C). (E) Schematic of the mARi-based repression system with high and low expression ratios constructed in single- and double-plasmid systems with different copy numbers. Reporter expression was measured for the single $(\mathbf{F})$ and double $(\mathbf{G})$ plasmid systems for high and low ratio $\mathrm{mARi}$ against a control construct without mARi; sfGFP fluorescence measurements were performed by flow cytometry assay. (H) The robustness of the double-plasmid mARibased regulation in different incubation temperatures. A schematic of the mARi-based repression system in double-plasmid systems used is illustrated. (I) The robustness of the double-plasmid mARi-based repression system in a variety of growth media. (J) Reporter expression of the single-plasmid high-ratio $\mathrm{mARi}$ on $\mathrm{p} 15 \mathrm{~A}$ was measured in different $E$. coli strains against a control construct without mARi. (K) The repression activity of single-plasmid high-ratio mARi on p15A in representative strains (DH10b, BL21 (DE3), and BL21star (DE3)) across different growth phases (Supplementary Figure S5). Assays in $\mathbf{H}, \mathbf{I}, \mathbf{J}$ and $\mathbf{K}$ were performed by plate reader assay. All error bars show the mean \pm SD of triplicate measurements (black dots). Statistically significant differences were determined against control without mARi expression (C) using two-tailed Student's $t$-test (**** represents $P<0.0001$, ${ }^{* * *}$ represents $P$ $<0.001,{ }^{* *}$ represents $P<0.01,{ }^{*}$ represents $P<0.1$, ns represents not significant). 
ferent levels of transcript abundance. These systems were first assembled with both mARi and its target in a single plasmid, with varying plasmid copy numbers from low to high: pSC101 ( $\sim 5$ copies per cell), p15A ( $\sim 10$ copies per cell) and pMB1 ( $\sim 15-20$ copies per cell) (55) (Figure 3E, left panel, Supplementary Figure S4a). Expression of mARi in either high or low expression ratio repressed sfGFP expression for all tested plasmid copy numbers, demonstrating that the mARi-based regulatory system is functional at different plasmid copy numbers (Figure $3 F$ ).

We next reasoned that by placing the mARi on a second, higher copy vector, repression should be more efficient. To test this hypothesis, the mRNA expression cassette was placed on a p15A backbone, while mARi was placed on the higher copy pMB1 and pUC backbones $(\sim 500-700$ copies per cell), with both high- and low-expression ratios (Figure 3E, right panel, Supplementary Figure S4b). In this experimental design, the transcript expression ratio was governed by the combination of plasmid copy number and transcription rate of mARi relative to mRNA. Despite mARi being driven by a stronger promoter and being on a higher copy vector, in all cases the double-plasmid systems all showed less repression than that observed with the corresponding single-plasmid systems (Figure 3G). This suggests a role for spatial organisation of transcripts in posttranscriptional repression, as anticipated from recent theoretical analysis (56). The fact that the sRNA-based regulator has a shorter half-life and stability than mRNA may further reduce the mARi effectiveness when it is used in a double plasmid system with a broader physical transcript distribution.

To investigate the robustness of mARi repression system, we further tested the double plasmid system with both high and low expression ratios in different temperatures and growth media. The repression activity of mARi in low expression ratios was not significantly affected by a combination of different growth temperatures and growth media (Figure 3H, I). However, a high expression ratio resulted in a slight increase of mARi repression activity in the use of higher incubation temperature and a defined growth medium.

\section{mARi-based regulation is robust to different genetic contexts}

Unlike other biomolecular regulators (e.g. transcription factors, TALEs, zinc fingers, or CRISPRi) $(5,57,58)$, mARi only requires transcriptional activity and endogenous $\mathrm{Hfq}$, which is autoregulated by Hfq binding its own mRNA (59). Thus, the expression of a short sequence for mARi would be expected to have a low cellular burden and not significantly impact host cell growth. To test this, the high transcript ratio for $\mathrm{mARi}$ in a $\mathrm{p} 15 \mathrm{~A}$ backbone and control plasmid without mARi expression were used to compare the effect of mARi overexpression on host fitness. We sought to test this repression system in four commonly used E. coli strains: DH $5 \alpha$, DH10b, BL21(DE3) and BL21star(DE3). The high expression of mARi did not affect bacterial growth across different host strains, indicating the mARi-based repression system has a low production cost, and does not impact significantly on host cells (Supplementary Figure S5).

We then sought to evaluate the silencing activity of mARi in these E. coli $\mathrm{K} 12$ and B strains. The mARi-based gene regulation utilises the native $E$. coli $\mathrm{Hfq}$ chaperone and degradosome complex (e.g. RNase E) as helper proteins to regulate gene repression. The intracellular abundance of these components is influenced by several factors, including the genotype and growth phase. For instance, the abundance of Hfq is constant during the exponential growth, but reduces when $E$. coli cells reach stationary phase (47,60-63).

Functional characterisation of mARi at early stationary phase, 8 h (Supplementary Figure S5), showed about 60$80 \%$ repression activity in both $E$. coli $\mathrm{K}-12$ and B strains (Figure 3J), while the silencing activity in the B strains was higher than in the K-12 strains for comparable plasmid copy numbers (Supplementary Figure S6). The mARi system was active across different phases of cell growth (Figure $3 \mathrm{~K}$ ): the E. coli $\mathrm{K}-12$ strain (DH10b cells) showed relatively stable repression activity in both mid-exponential and early stationary growth, whereas a slightly increased repression activity was observed in early stationary phase for the BL21(DE3) and BL21star(DE3) strains.

\section{Expanding a set of orthogonal $\mathrm{mARi}$ regulators through seed sequence modification}

We sought to create a set of mARi-based regulators based on its underlying modular design principles coupled with the modular UTR-RBS linkers used in BASIC to construct both expression cassettes and operons. Five different UTRRBS linkers were identified using R2oDNA Designer (35), whilst preserving the same medium strength RBS (RBSc). Computational evaluation of the five selected UTR (UTRA-E) sequences demonstrated minimal similarity/identity scores (Supplementary Figure S7a) and high Hamming distance scores (Supplementary Figure S7b) between the set. Low sequence similarity and high Hamming distance of non-cognate pairs is desirable to achieve minimal crosstalk interaction and off-target effects (30). The target 35 bp upstream of the RBS sequence were evaluated for their GC content and free binding energy of the target/seed sequences (Supplementary Table S4). The GC content of the seed sequence is in a range of $31 \%$ to $43 \%$ and resulted in a free binding energy of -53.9 to $-63.9 \mathrm{kcal} / \mathrm{mol}$, which were in the favourable range for sRNA repression $(30,31)$. The seed sequences were also predicted to have minimal off-target interactions with three different $E$. coli genomes: E. coli MG1655, DH10b, and BL21(DE3) (Supplementary Table S4).

To experimentally validate target specificity, we constructed all possible combinations of $\mathrm{mARi}$ and target UTR upstream of $s f g f p$; in each case maintaining the same promoter (Figure 4A). The expression of sfGFP was significantly repressed in the presence of the cognate mARi systems relative to the non-cognate pairings (Figure 4A, Supplementary Figure S7d), confirming their specificity with reduced cross-reactivity between each pair. However, there is an increased expression in UTR-D-sfGFP when mARi-A and mARi-B were used. The lower Hamming score of UTR D towards UTR A (Supplementary Figure S7b) may contribute to this, but it does not completely explain the effect with mARi-B, or other observations; similar effects were not observed for UTR-E, which similarly has lower Hamming scores for UTR-A, -B and -D (Supplementary Figure S7b). 
A

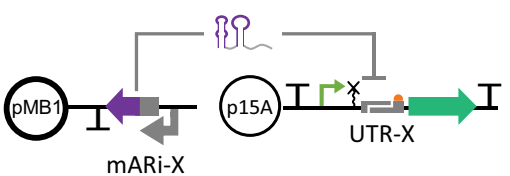

UTR-A UTR-B UTR-C UTR-D UTR-E

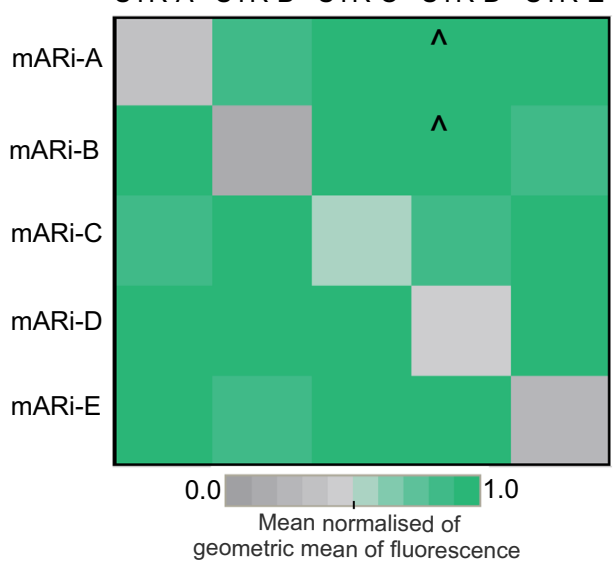

B
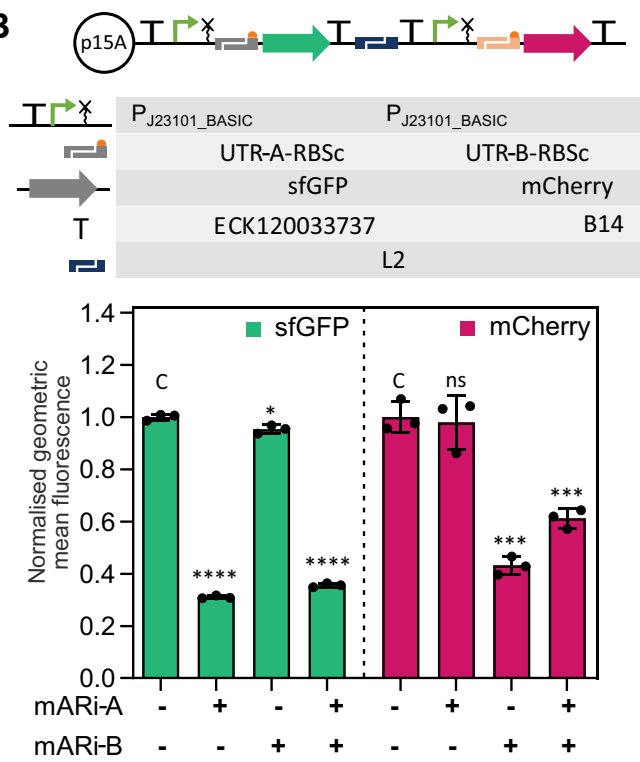
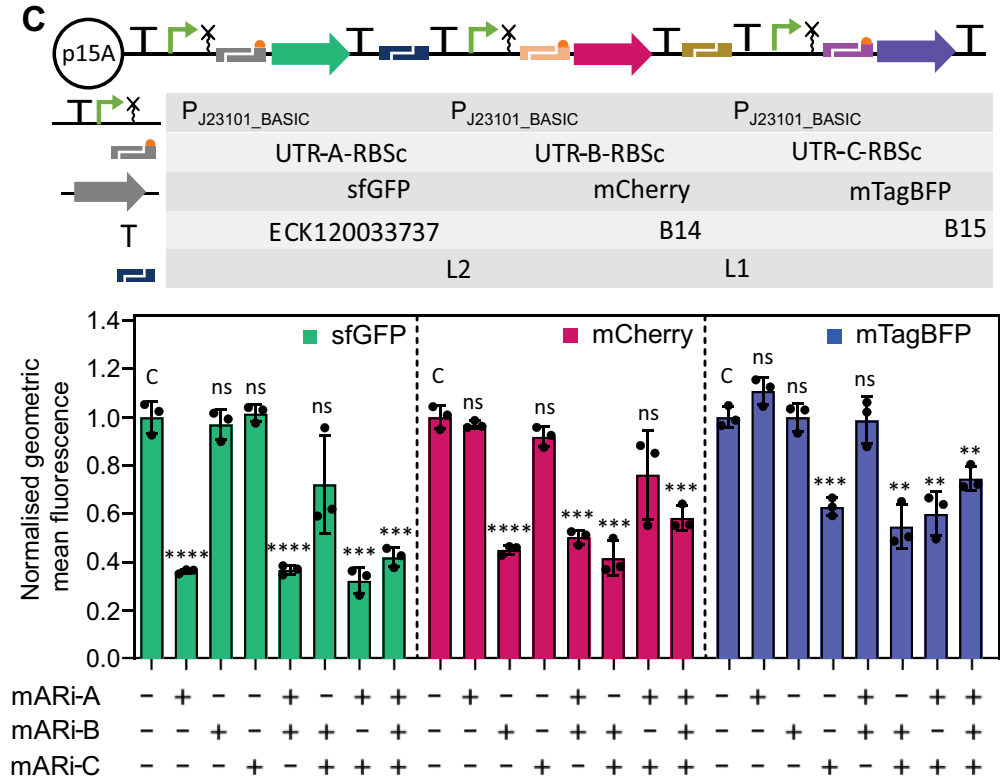

D
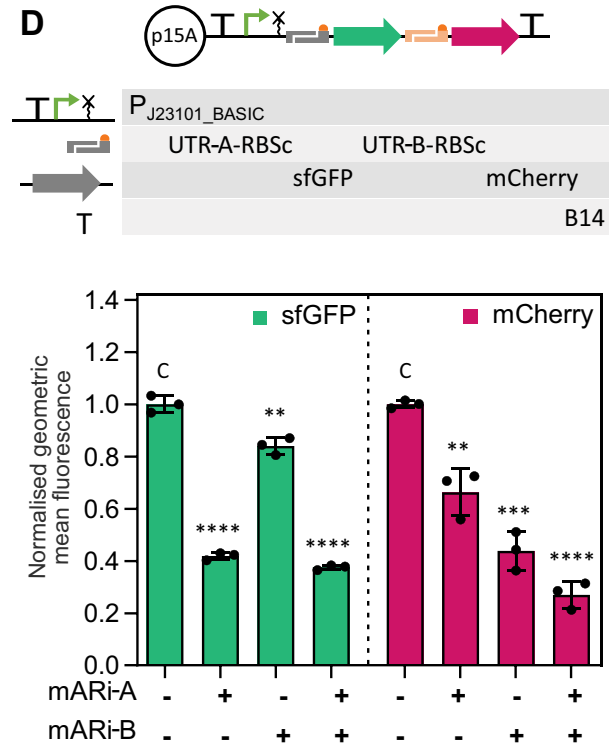

Figure 4. mARi enables multiplex and simultaneous control of gene expression in a multi-gene system. (A) A schematic of experimental design to evaluate the target specificity of modular mARi-mRNA pairs is shown. Fluorescence response matrix of all possible mARi/UTR pairs measured by flow cytometry assay with triplicate repeats (Supplementary Figure S7d); ${ }^{\wedge}$ represents data where the use of mARi-A and mARi-B increased observed UTR-D-sfGFP expression relative to control. (B) A schematic of reporters in dual transcription units is shown: the fluorescence response of reporters in the double transcriptional unit system when combined with all possible mARi-A and mARi-B regulators was measured by flow cytometry assay. (C) A schematic of reporters in triple transcription units is shown: the fluorescence response of reporters in the triple transcriptional unit system when combined with all possible mARi-A, mARi-B and mARi-C regulators was measured by flow cytometry assay. (D) A schematic of dual reporters in an operon system is shown: the fluorescence response of dual reporters in the operon system when combined with all possible mARi-A and mARi-B regulators was measured by flow cytometry assay. All error bars show the mean \pm SD of triplicate measurements (black dots). Statistically significant differences were determined against control without mARi expression $(\mathbf{C})$ using two-tailed Student's $t$-test $(* * * *$ represents $P<0.0001, * * *$ represents $P<0.001, * *$ represents $P<$ $0.01, *$ represents $P<0.1$, ns represents not significant).

\section{Modularity and orthogonality of mARi enables simultaneous and multiplexed gene regulation}

After demonstrating that mARi regulators exhibit specific interaction towards cognate UTRs in the context of a single gene, we evaluated their ability to operate in a multiplexed reporter gene design. Initially, this was evaluated with both reporter genes (i.e. $s f g f p$ and $m c h e r r y$ ) organised as separate transcriptional units (Figure 4B). Specific and independent repression by each mARi towards its cognate target was observed, with UTR-A-sfgfp being selectively targeted by mARi-A and UTR-B-mcherry being targeted by mARi-B (Figure 4B and Supplementary Figure S8a). Further, specific and independent repression was also confirmed when three mARi regulators (mARi-A, mARi-B, and mARi-C) were employed to target UTR-A, UTR-B, and UTR-C pre- 
ceding the $s f g f p$, mcherry, and mtagbfp genes, respectively (Figure 4C and Supplementary Figure S8b).

Two sets of mARi regulators were used to modulate gene expression in a two-gene operon with UTR-A-RBSc driving the expression of $s f g f p$, upstream of UTR-B-RBSc driving the expression of mcherry, constitutively expressed by the promoter $\mathrm{P}_{\mathrm{J} 23101 \_B A S I C}($ Figure $4 \mathrm{D})$ ). With both genes on a single transcript, the targeted repression for the first gene $(s f g f p)$ is strongly affected by its cognate mARi-A, with only a minor affect from $\mathrm{mARi}-\mathrm{B}$, which targets the downstream mcherry; the downstream mcherry is most strongly attenuated by its cognate mARi-B, but it is also significantly attenuated by repression of the upstream $s f g f p$ with full attenuation only reached with both $\mathrm{mARi}-\mathrm{A}$ and B (Figure 4D and Supplementary Figure S8c).

\section{DISCUSSION}

Here, we report the development, characterisation, and implementation of a modular post-transcriptional regulation system based on trans-encoded sRNAs. The modular design of mARi is derived from the highly composable structure of the natural MicC sRNAs scaffold $(17,27)$. A potential limitation of our system is that our mARi regulators utilise shared endogenous Hfq chaperone for their repression activity. However, endogenous Hfq is autoregulated by Hfq binding its own mRNA, even when expressed from plasmid-borne genes (59) and the intracellular concentration of available Hfq can adapt rapidly to cellular changes in the RNA pool by facilitated recycling of the protein (64). In addition, deviation from the optimum $\mathrm{Hfq}$ concentration can lead to suppression of sRNA activity in E. coli (29). Efforts to modulate sRNA activity by exogenous expression of Hfq would therefore require careful balancing of the whole system.

Modification of the seed sequence, whilst retaining the sRNA scaffold, has enabled the reprogramming of natural sRNAs with different targets of interests that do not exist in nature $(17,18,28)$. Here, we utilised this modularity for targeted gene repression by directing mARi regulators to standardised UTRs in the translation initiation region of the mRNA target. We demonstrated that targeted repression by binding to standardised sequences in the 5'-UTR upstream of the RBS were independent from both RBS and GOI contexts. The computationally designed orthogonal UTR sequences of the BASIC UTR-RBS linkers proved to be ideal target sites for mARi regulators (Figures 2C, E and 4). The standardised linker sequences that define the UTR have been computationally generated and validated to ensure their orthogonality in the DNA assembly process, as well as to the $E$. coli host (35). Therefore, by targeting the BASIC DNA linkers, it was anticipated that the mARi would exert orthogonal, post-transcriptional gene regulation with minimal cross-interaction to non-cognate targets, bioparts, plasmid backbones and host genotypes (35). The sequence composition of seed/target sites can be refined to optimise their binding affinity. This approach may result in higher repression and orthogonality. Therefore, it could also be coupled with an improved algorithm to create an sRNA optimised orthogonal sequence for the assembly process using BASIC DNA assembly.
The mARi-based repression of a polycistronic mRNA provides some insight into its mechanism of action. Facilitated degradation of the mRNA would be expected to induce a similar repression level on both genes, even when only one is targeted, but single-gene targeting in an operon led to a differential effect between the operon's genes. A non-targeted gene downstream of the mARi gene target exhibited significant repression, while a non-targeted gene upstream of the mARi gene target exhibited only slight repression (Figure 4D). This behaviour is consistent with steric hindrance of the ribosome for the UTR-RBS preventing translation initiation being the main mode of action of the mARi sRNA, rather than active degradation. The silencing effects on the downstream mcherry gene were also additive for both the gene's cognate mARi and the upstream gene's $\mathrm{mARi}$, suggesting that repression at each UTR-RBS position was independent. It further suggests that read-through was possible from the upstream gene, even when the downstream RBS was repressed, but this was occluded when both UTRs were bound by their respective mARi regulators.

The mechanism of action being based on the inhibition of translation initiation rather than active degradation may also be due to the designed mARi regulators and UTRRBS linkers omitting an RNase E binding site, which is essential for RNase E-dependent cleavage. The elimination of RNase $\mathrm{E}$ in the $\mathrm{mARi}$ design was anticipated to reduce background degradation of unpaired mARi regulators and improve their cellular half-lives. The exclusion of an RNase E cleavage site in the mARi design may be beneficial for the implementation of an orthogonal repression system in various $E$. coli strains, with a response that is independent of the host genotype. Indeed, the system was observed to function across different $E$. coli strains and growth phases, demonstrating both its portability and robustness, notably with minimal difference between BL21(DE3) and BL21star(DE3), the latter being deficient in RNase E (Figure 3).

The modular nature of the design framework meant facile variation of genetic design parameters. Our observations of design characteristics were consistent with previous studies (30), where repression increased as a function of length and hence free binding energy (Supplementary Figure S2). Importantly, we have also identified spatial organisation and relative transcript abundance as being critical factors in efficient repression by sRNA (Figure 3). Expressing mARi in a high expression ratio and a single plasmid system with medium plasmid copy number (p15A) resulted in up to $80 \%$ repression activity. With trans expression of mARi from a second plasmid, the repression ratio was always worse, even when it was encoded on a higher plasmid copy number. Also somewhat surprising was the low efficiency of repression in a low-copy (pSC101) single plasmid system. This likely represents the importance of having the correct balance of all components relative to the native Hfq availability, as anticipated from Sagawa et al. (29). The importance of spatial organisation is somewhat surprising given the abundance of natural trans-encoded systems, but it is likely to originate from enhanced proximity and hence binding between the mARi and its target.

The modular nature of the mARi design enabled control of different genes and facilitated multiplexing of target 
genes. In total, five pairs of orthogonal mARi/UTR regulators were created and tested in this work. These mARi regulators were applied to multiplexed and simultaneous posttranscriptional regulation in multi-gene systems, including both multiple transcriptional units and an operon architecture (Figure 4). Importantly, the orthogonal basis of the BASIC design framework removed the need to design or insert bespoke target sites upstream of target genes, a drawback of previous works focused on reusable trans-encoded small RNAs $(7,24-26,28)$; it was implemented by simply assembling the mARi regulators and their cognate targets using BASIC DNA assembly without re-optimisation of the regulator for different target genes. This extensibility is essential for the simultaneous regulation of multiple genes in metabolic engineering, layered genetic control, and advanced genetic circuit applications, while scalability is possible through our recently demonstrated low cost automation platform for BASIC assembly (33).

\section{DATA AVAILABILITY}

The data underlying this article will be shared on reasonable reuest to the corresponding author.

\section{SUPPLEMENTARY DATA}

Supplementary Data are available at NAR Online.

\section{ACKNOWLEDGEMENTS}

Author contributions: A.D.: Designed and performed all experiments and data analysis, devised the experimental strategy and wrote the manuscript. M.S.: Devised experimental strategy and design, assisted with the experiments and data interpretation and wrote the manuscript. G.-B.S.: Contributed to the experimental strategy, assisted with experimental design and data interpretation and wrote the manuscript. G.S.B.: Contributed to the experimental strategy, assisted with the experimental design and data interpretation and wrote the manuscript.

\section{FUNDING}

A.D. received a $\mathrm{PhD}$ scholarship from Indonesia Endowment Fund for Education (LPDP); G.-B.S. gratefully acknowledges the support of the UK EPSRC through the EPSRC Fellowship for Growth [EP/M002187/1] and of the Royal Academy of Engineering through the Chair in Emerging Technology programme; G.S.B. acknowledges the support of UK Research and Innovation through the Engineering and Physical Sciences Research Council [EP/R034915/1]; EU through H2020 [820699]. Funding for open access charge: A.D. received a PhD scholarship from Indonesia Endowment Fund for Education (LPDP); G.-B.S. gratefully acknowledges the support of the UK EPSRC through the EPSRC Fellowship for Growth [EP/M002187/1] and of the Royal Academy of Engineering through the Chair in Emerging Technology programme; G.S.B. acknowledges the support of UK Research and Innovation through the Engineering and Physical Sciences Research Council [EP/R034915/1]; EU through H2020 [820699].
Conflict of interest statement. None declared.

\section{REFERENCES}

1. Cameron,D.E., Bashor,C.J. and Collins,J.J. (2014) A brief history of synthetic biology. Nat. Rev. Microbiol., 12, 381-390.

2. Purnick,P.E.M. and Weiss,R. (2009) The second wave of synthetic biology: from modules to systems. Nat. Rev. Mol. Cell Biol., 10, $410-422$.

3. Slusarczyk,A.L., Lin,A. and Weiss,R. (2012) Foundations for the design and implementation of synthetic genetic circuits. Nat. Rev. Genet., 13, 406-420.

4. Kelwick,R., MacDonald,J.T., Webb,A.J. and Freemont,P. (2014) Developments in the tools and methodologies of synthetic biology. Front. Bioeng. Biotechnol., 2, 60.

5. Xiang,Y., Dalchau,N. and Wang,B. (2018) Scaling up genetic circuit design for cellular computing: advances and prospects. Nat. Comput., 17, 833-853.

6. Storz,G., Vogel,J. and Wassarman,K.M. (2011) Regulation by small RNAs in bacteria: expanding frontiers. Mol. Cell, 43, 880-891.

7. Huang,H.-H., Qian,Y. and Del Vecchio,D. (2018) A quasi-integral controller for adaptation of genetic modules to variable ribosome demand. Nat. Commun., 9, 5415.

8. Thompson,K.M., Rhodius,V.A. and Gottesman,S. (2007) $\sigma \mathrm{E}$ regulates and is regulated by a small RNA in Escherichia coli. $J$. Bacteriol., 189, 4243-4256.

9. Tu,K.C., Long,T., Svenningsen,S.L., Wingreen,N.S. and Bassler,B.L. (2010) Negative feedback loops involving small regulatory RNAs precisely control the vibrio harveyi quorum-sensing response. Mol. Cell, 37, 567-579.

10. Brosse,A., Korobeinikova,A., Gottesman,S. and Guillier,M. (2016) Unexpected properties of sRNA promoters allow feedback control via regulation of a two-component system. Nucleic Acids Res., 44, 9650-9666.

11. Nitzan,M., Rehani,R. and Margalit,H. (2017) Integration of bacterial small RNAs in regulatory networks. Annu. Rev. Biophys., 46, 131-148.

12. Beisel,C.L. and Storz,G. (2010) Base pairing small RNAs and their roles in global regulatory networks. FEMS Microbiol. Rev., 34, $866-882$.

13. Levine,E., Zhang,Z., Kuhlman,T. and Hwa,T. (2007) Quantitative characteristics of gene regulation by small RNA. PLoS Biol., 5 , 1998-2010.

14. Rice,J.B., Balasubramanian,D. and Vanderpool,C.K. (2012) Small RNA binding-site multiplicity involved in translational regulation of a polycistronic mRNA. Proc. Natl. Acad. Sci., 109, E2691-E2698.

15. Morita,T. and Aiba,H. (2011) RNase e action at a distance: degradation of target mRNAs mediated by an Hfq-binding small RNA in bacteria. Genes Dev., 25, 294-298.

16. Pfeiffer,V., Papenfort,K., Lucchini,S., Hinton,J.C.D. and Vogel,J. (2009) Coding sequence targeting by MicC RNA reveals bacterial mRNA silencing downstream of translational initiation. Nat. Struct. Mol. Biol., 16, 840-846.

17. Yoo,S.M., Na,D. and Lee,S.Y. (2013) Design and use of synthetic regulatory small RNAs to control gene expression in Escherichia coli. Nat. Protoc., 8, 1694-1707.

18. Sharma,V., Yamamura,A. and Yokobayashi,Y. (2012) Engineering artificial small RNAs for conditional gene silencing in Escherichia coli. ACS Synth. Biol., 1, 6-13.

19. Viegas,S.C., Silva,I.J., Saramago,M., Domingues,S. and Arraiano,C.M. (2011) Regulation of the small regulatory RNA MicA by ribonuclease III: a target-dependent pathway. Nucleic Acids Res., 39, 2918-2930.

20. Man,S., Cheng,R., Miao,C., Gong,Q., Gu,Y., Lu,X., Han,F. and Yu,W. (2011) Artificial trans-encoded small non-coding RNAs specifically silence the selected gene expression in bacteria. Nucleic Acids Res., 39, e50.

21. Nakashima,N., Tamura,T. and Good,L. (2006) Paired termini stabilize antisense RNAs and enhance conditional gene silencing in Escherichia coli. Nucleic Acids Res., 34, e138.

22. Engdahl,H.M., Lindell,M. and Wagner,E.G.H. (2001) Introduction of an RNA stability element at the $5^{\prime}$-End of an antisense RNA cassette increases the inhibition of target RNA translation. Antisense Nucleic Acid Drug Dev., 11, 29-40. 
23. Ellis,M.J., Trussler,R.S. and Haniford,D.B. (2015) A cis-encoded sRNA, hfq and mRNA secondary structure act independently to suppress IS200 transposition. Nucleic Acids Res., 43, 6511.

24. Ghodasara,A. and Voigt,C.A. (2017) Balancing gene expression without library construction via a reusable sRNA pool. Nucleic Acids Res., 45, 8116-8127.

25. Kelly,C.L., Harris,A.W.K., Steel,H., Hancock,E.J., Heap,J.T. and Papachristodoulou,A. (2018) Synthetic negative feedback circuits using engineered small RNAs. Nucleic Acids Res., 46, 9875-9889.

26. Ceroni,F., Furini,S., Stefan,A., Hochkoeppler,A. and Giordano,E. (2012) A synthetic post-transcriptional controller to explore the modular design of gene circuits. ACS Synth. Biol., 1, 163-171.

27. Na,D., Yoo,S.M., Chung,H., Park,H., Park,J.H. and Lee,S.Y. (2013) Metabolic engineering of Escherichia coli using synthetic small regulatory RNAs. Nat. Biotechnol., 31, 170-174.

28. Lahiry,A., Stimple,S.D., Wood,D.W. and Lease,R.A. (2017) Retargeting a dual-acting sRNA for multiple mRNA transcript regulation. ACS Synth. Biol., 6, 648-658.

29. Sagawa,S., Shin,J.-E., Hussein,R. and Lim,H.N. (2015) Paradoxical suppression of small RNA activity at high hfq concentrations due to random-order binding. Nucleic Acids Res., 43, 8502-8515.

30. Hoynes-O'Connor,A. and Moon,T.S. (2016) Development of design rules for reliable antisense RNA behavior in e. coli. ACS Synth. Biol., 5, 1441-1454.

31. Lee,Y.J. and Moon,T.S. (2018) Design rules of synthetic non-coding RNAs in bacteria. Methods, 143, 58-69.

32. Massaiu,I., Pasotti,L., Casanova,M., Politi,N., Zucca,S., Cusella De Angelis,M.G. and Magni,P. (2015) Quantification of the gene silencing performances of rationally-designed synthetic small RNAs. Syst. Synth. Biol., 9, 107-123.

33. Storch,M., Haines,M.C. and Baldwin,G.S. (2020) DNA-BOT: a low-cost, automated DNA assembly platform for synthetic biology. Synth. Biol., 5, ysaa010.

34. Storch,M., Casini,A., Mackrow,B., Fleming,T., Trewhitt,H., Ellis,T. and Baldwin,G.S. (2015) BASIC: a new biopart assembly standard for idempotent cloning provides accurate, single-tier DNA assembly for synthetic biology. ACS Synth. Biol., 4, 781-787.

35. Casini,A., Christodoulou,G., Freemont,P.S., Baldwin,G.S., Ellis,T. and MacDonald,J.T. (2014) R2oDNA designer: computational design of biologically neutral synthetic DNA sequences. ACS Synth. Biol., 3, 525-528.

36. Darty,K., Denise,A. and Ponty,Y. (2009) VARNA: interactive drawing and editing of the RNA secondary structure. Bioinformatics, 25, 1974-1975.

37. Busch,A., Richter,A.S. and Backofen,R. (2008) IntaRNA: efficient prediction of bacterial sRNA targets incorporating target site accessibility and seed regions. Bioinformatics, 24, 2849-2856.

38. Wright,P.R., Georg,J., Mann,M., Sorescu,D.A., Richter,A.S., Lott,S., Kleinkauf,R., Hess,W.R. and Backofen,R. (2014) CopraRNA and intarna: predicting small RNA targets, networks and interaction domains. Nucleic Acids Res., 42, 119-123.

39. Markham,N.R. and Zuker,M. (2005) DINAMelt web server for nucleic acid melting prediction. Nucleic Acids Res., 33, 577-581.

40. Bonde,M.T., Pedersen,M., Klausen,M.S., Jensen,S.I., Wulff,T., Harrison,S., Nielsen,A.T., Herrgård,M.J. and Sommer,M.O.A. (2016) Predictable tuning of protein expression in bacteria. Nat Methods, 13, 233-236.

41. Salis,H.M., Mirsky,E.A. and Voigt,C.A. (2009) Automated design of synthetic ribosome binding sites to control protein expression. Nat. Biotechnol., 27, 946-950.

42. Espah Borujeni,A., Channarasappa,A.S. and Salis,H.M. (2014) Translation rate is controlled by coupled trade-offs between site accessibility, selective RNA unfolding and sliding at upstream standby sites. Nucleic Acids Res., 42, 2646-2659.

43. Raden,M., Ali,S.M., Alkhnbashi,O.S., Busch,A., Costa,F., Davis,J.A., Eggenhofer,F., Gelhausen,R., Georg,J., Heyne,S. et al. (2018) Freiburg RNA tools: a central online resource for
RNA-focused research and teaching. Nucleic Acids Res., 46, W25-W29.

44. Wright,P.R., Richter,A.S., Papenfort,K., Mann,M., Vogel,J., Hess,W.R., Backofen,R. and Georg,J. (2013) Comparative genomics boosts target prediction for bacterial small RNAs. Proc. Natl. Acad. Sci. U.S.A., 110, E3487-E3496.

45. Storch,M., Casini,A., Mackrow,B., Ellis,T. and Baldwin,G.S. (2017) BASIC: a simple and accurate modular DNA assembly method. In: Methods in Molecular Biology. Vol. 1472, pp. 79-91.

46. Vytvytska,O., Moll,I., Kaberdin,V.R., Von Gabain,A. and Bläsi,U. (2000) Hfq (HF1) stimulates ompA mRNA decay by interfering with ribosome binding. Genes Dev., 14, 1109-1118.

47. Večerek,B., Moll,I. and Bläsi,U. (2005) Translational autocontrol of the escherichia coli hfq RNA chaperone gene. RNA, 11, 976-984

48. Franze De Fernandez,M.T., Eoyang,L. and August,J.T. (1968) Factor fraction required for the synthesis of bacteriophage Qß-RNA. Nature, 219, 588-290.

49. Sun,T., Li,S., Song,X., Pei,G., Diao,J., Cui,J., Shi,M., Chen,L. and Zhang,W. (2018) Re-direction of carbon flux to key precursor malonyl-CoA via artificial small RNAs in photosynthetic synechocystis sp. PCC 6803. Biotechnol. Biofuels, 11, 26.

50. Huis in 't Veld,R.A.G., Kramer,G., van der Ende,A., Speijer,D. and Pannekoek, Y. (2017) The hfq regulon of neisseria meningitidis. FEBS Open Bio, 7, 777-788.

51. Puerta-Fernández,E. and Vioque,A. (2011) Hfq is required for optimal nitrate assimilation in the cyanobacterium anabaena sp. strain PCC 7120. J. Bacteriol., 193, 3546-3555.

52. Chen,S., Zhang,A., Blyn,L.B. and Storz,G. (2004) MicC, a second Small-RNA regulator of omp protein expression in escherichia coli MicC, a second Small-RNA regulator of omp protein expression in escherichia coli. J. Bacteriol., 186, 6689-6697.

53. Vogel,J. and Luisi,B.F. (2011) Hfq and its constellation of RNA. Nat. Rev. Microbiol., 9, 578-589.

54. Mitarai,N., Benjamin,J.-A.M.J.-A.M., Krishna,S., Semsey,S., Csiszovszki,Z., Massé,E., Sneppen,K., Masse,E. and Sneppen,K. (2009) Dynamic features of gene expression control by small regulatory RNAs. Proc. Natl. Acad. Sci. U.S.A., 106, 10655-10659.

55. Jahn,M., Vorpahl,C., Hübschmann,T., Harms,H. and Müller,S. (2016) Copy number variability of expression plasmids determined by cell sorting and droplet digital PCR. Microb. Cell Fact., 15, 211.

56. Teimouri,H., Korkmazhan,E., Stavans,J. and Levine,E. (2017) Sub-cellular mRNA localization modulates the regulation of gene expression by small RNAs in bacteria. Phys. Biol., 14, 056001

57. Nielsen,A.A.K.K., Segall-shapiro,T.H. and Voigt,C.A. (2013) Advances in genetic circuit design: novel biochemistries, deep part mining, and precision gene expression. Curr. Opin. Chem. Biol., 17, 878-892.

58. Bradley,R.W., Buck,M. and Wang,B. (2016) Tools and principles for microbial gene circuit engineering. J. Mol. Biol., 428, 862-888.

59. Morita,T. and Aiba,H. (2019) Mechanism and physiological significance of autoregulation of the escherichia coli hfa gene. $R N A$, 25, 264-276.

60. Azam,T.A., Iwata,A., Nishimura,A., Ueda,S. and Ishihama,A. (1999) Growth phase-dependent variation in protein composition of the escherichia coli nucleoid. J. Bacteriol., 181, 6361-6370.

61. Kajitani,M., Kato,A., Wada,A., Inokuchi,Y. and Ishihama,A. (1994) Regulation of the escherichia coli hfq gene encoding the host factor for phage $\mathrm{Q}(\beta)$. J. Bacteriol., 176, 531-534.

62. Yoon,S., Han,M.-J., Jeong,H., Lee,C., Xia,X.-X., Lee,D.-H., Shim,J., Lee,S., Oh,T. and Kim,J.F. (2012) Comparative multi-omics systems analysis of Escherichia coli strains B and K-12. Genome Biol., 13, R37.

63. Moon,K. and Gottesman,S. (2011) Competition among Hfq-binding small RNAs in Escherichia coli. Mol. Microbiol., 82, 1545-1562.

64. Wagner,E.G.H. (2013) Cycling of RNAs on hfq. RNA Biol., 10, 619-626. 